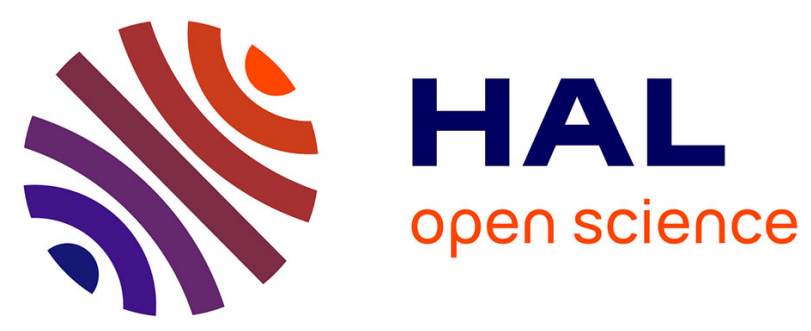

\title{
Distributed Brillouin Fiber Sensor With Enhanced Sensitivity Based on Anti-Stokes Single-Sideband Suppressed-Carrier Modulation
}

Duc Minh Nguyen, Birgit Stiller, Min Won Lee, Jean-Charles Beugnot, Hervé Maillotte, Alexandre Mottet, Jérôme Hauden, Thibaut Sylvestre

\section{To cite this version:}

Duc Minh Nguyen, Birgit Stiller, Min Won Lee, Jean-Charles Beugnot, Hervé Maillotte, et al.. Distributed Brillouin Fiber Sensor With Enhanced Sensitivity Based on Anti-Stokes Single-Sideband Suppressed-Carrier Modulation. IEEE Photonics Technology Letters, 2013, 25 (1), pp.94-96. 10.1109/LPT.2012.2228848 . hal-01610084

\section{HAL Id: hal-01610084 \\ https://hal.science/hal-01610084}

Submitted on 4 Oct 2017

HAL is a multi-disciplinary open access archive for the deposit and dissemination of scientific research documents, whether they are published or not. The documents may come from teaching and research institutions in France or abroad, or from public or private research centers.
L'archive ouverte pluridisciplinaire HAL, est destinée au dépôt et à la diffusion de documents scientifiques de niveau recherche, publiés ou non, émanant des établissements d'enseignement et de recherche français ou étrangers, des laboratoires publics ou privés. 


\title{
Distributed Brillouin Fiber Sensor with Enhanced Sensitivity based on Anti-Stokes Single-Sideband Suppressed-Carrier Modulation
}

\author{
D. M. Nguyen, B. Stiller, M. W. Lee, J. C. Beugnot, H. Maillotte, A. Mottet, J. Hauden, and T. Sylvestre
}

\begin{abstract}
We demonstrate an alternative technique to reduce pump depletion and to improve sensitivity in long-range Brillouin optical time-domain analysis (BOTDA) sensor. Our BOTDA system uses an anti-Stokes single-sideband suppressed-carrier (SSB-SC) modulation technique that balances the pump depletion due to fiber loss. With this technique, we show both numerically and experimentally a great improvement compared to the dual sideband standard technique.
\end{abstract}

Index Terms-Fiber optic sensor, Brillouin optical time domain analysis, single-sideband modulation.

\section{INTRODUCTION}

B RILLOUIN Optical Time-Domain Analysis (BOTDA) sensing systems have been the subject of intense research in recent years because they offer a unique solution for continuous, real-time monitoring in civil or geotechnical engineering and petroleum industry. These fiber-optic sensors provide distributed strain or temperature measurements with meter spatial resolution over several tens of kilometers. An important factor that limits the range of BOTDA sensors however is pump depletion. This non-local effect degrades the signal-to-noise ratio (SNR) and distorts the Brillouin gain spectrum (BGS), leading to systematic errors or biases in sensors measurements [1]- [3]. Several techniques have recently been proposed to overcome these limitations and to extend the sensing range beyond $50 \mathrm{~km}$. For instance, the sensing range can be extended to $100 \mathrm{~km}$ by using distributed Raman amplification schemes [4], or $150 \mathrm{~km}$ by combining frequency-division multiplexing and in-line Erbium doped fiber amplifiers (EDFAs) [5]. 120-km range has also been achieved by improving the SNR with optical pulse coding techniques and optical pre-amplification at the receiver [6]. All of these techniques rely on the use a dual sideband (DSB) probe which reduces the impact of pump depletion. Recently, Bernini et al. [7] proposed a BOTDA system making use of an unbalanced DSB probe with a Stokes / anti-Stokes power ratio allowing a larger suppression of nonlocal effects induced by pump depletion.

In this paper, we describe and demonstrate an alternative

D.M. Nguyen, B. Stiller, M. W. Lee, J. -C. Beugnot, H. Maillotte and T. Sylvestre are with FEMTO-ST institute, Universit de Franche-Comt, UMR CNRS 6174, 25030 Besancon, France (e-mail: thibaut.sylvestre@univfcomte.fr, ducminh.nguyen@femto-st.fr).

A. Mottet and J. Hauden are with PHOTLINE Technologies, 25000 Besancon, France.

This work was financed by the European program INTERREG IVA (FEDER) and the region Franche-Comté.

Manuscript received July 9, 2012.
Brillouin-loss based technique to further reduce pump depletion and to improve the sensitivity in BOTDA systems. Compared to the technique of Bernini [7], our BOTDA system uses an anti-Stokes probe generated with a single-sideband suppressed-carrier (SSB-SC) modulator to balance the pump depletion due to fiber loss [8]. With this technique, we show both numerically and experimentally enhanced performances compared to conventional DSB schemes. Distributed strain measurements over a $50-\mathrm{km}$ of single-mode fiber (SMF) with $3 \mathrm{~m}$ spatial resolution are reported.

\section{NUMERICAL SIMULATIONS}

Let us consider the stimulated Brillouin scattering (SBS) between a square pump pulse and two counter-propagating continuous-wave (CW) Stokes and anti-Stokes waves. At each fiber length, the interaction time is given by the pump pulse duration if it is sufficiently longer than the phonon lifetime $(\sim 10 \mathrm{~ns})$. During the interaction, the pump will transfer its energy to the Stokes probe (loss process) and also receive energy from the anti-Stokes beam (gain process). The gain and loss process of the pump correspond respectively to the Brillouin loss and gain for the signal. This gain/loss process is not linear since the Stokes and anti-Stokes powers change during the interaction time. This results in a non-uniform deformation of the pump that cannot be ignored in long-range measurements. To get better insight, we performed numerical simulations based on three coupled equations for the pump, Stokes and anti-Stokes waves using the finite different method (for equations, see [9]). The input pump pulse duration is 500 ns and its peak power is $0.1 \mathrm{~W}$. All other parameters are listed in the caption of Fig. 1. We also restricted the sensing length to $2.8 \mathrm{~km}$ in the numerics because taking into account a $50-\mathrm{km}$ value as in experiment is very time-consuming and computationally intensive. Fig. 1(a) shows the computed pump pulse at the output of the sensing fiber for different input probe conditions, as indicated in the inset and in the caption. More specifically, we compare the Stokes and anti-Stokes SSB probe regimes with the balanced and unbalanced DSB ones. As it can be seen, in the Stokes SSB regime, the output pump pulse is strongly distorted and depleted (solid green curve) compared to the pump alone without input probe (dashed blue curve). The output pump power can however be raised by using the DSB regime, as shows the dashed-dotted pulse in Fig. 1(a). In this regime, the anti-Stokes sideband strengthens the pump energy through the gain process that reduces pump depletion. 

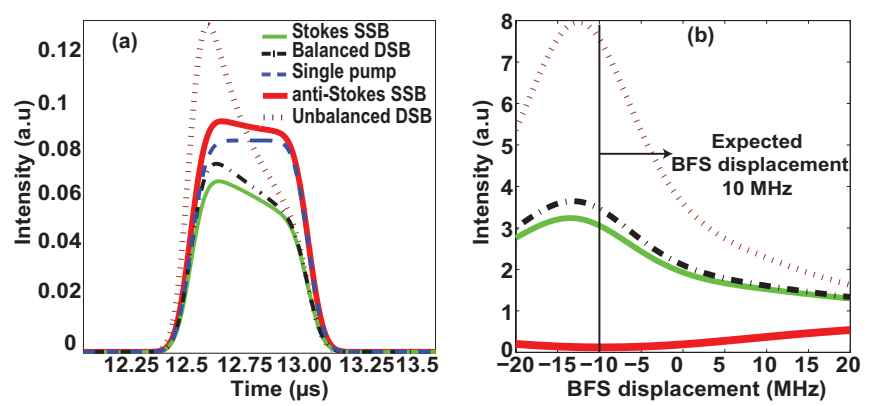

Fig. 1. Numerical simulations of (a) the pump pulse depletion and (b) the probe intensity versus the strain-induced BFS displacement for 5 different input conditions of the Stokes/anti-Stokes power ratio. Stokes power is 500 $\mu \mathrm{W}$ in Stokes SSB, balanced DSB and unbalanced DSB regimes. Anti-Stokes power is $500 \mu \mathrm{W}$ in the balanced DSB and the anti-Stokes SSB regimes. In the unbalanced DSB regime, the anti-Stokes power is $3.5 \mathrm{~mW}$. Parameters are fiber length $L=2.8 \mathrm{~km}$, Brillouin gain $g=1 \cdot 66 \cdot 10^{-11} \mathrm{~m} . \mathrm{W}^{-1}$, loss coefficient $\alpha=0.2 \mathrm{~dB} / \mathrm{km}$.

Nevertheless, pump depletion still remains in the balanced DSB case. This is due to the fact that energy transfer from the pump to the Stokes probe is faster than from the anti-Stokes probe to the pump once the Stokes power exceeds that of the anti-Stokes one. In the unbalanced DSB regime (dotted red curve), the pump peak power reaches a high value but its shape and width are strongly modified by the Brillouin interaction. The solid red curve shows a direct comparison with our technique making use of a single-sideband anti-Stokes probe. As it can be seen, the pump pulse is no longer depleted and its shape is the closest to the initial one shown in blue. In such a regime, the pump power is depleted only due to the fiber loss. This leads to a great improvement of the output pump peak power and pulse shape, compared to the other probe regimes. We further compared these different probe conditions when applying strain on the fiber in order to assess the possible systematic errors induced by pump depletion for the Brillouin frequency shift (BFS). Fig. 1(b) shows the computed values of strain-induced BFS displacement. This numerical simulation was done with an assumption of an intrinsic SBS line-width of $35 \mathrm{MHz}$ and a strain applied at the end of the fiber of $200 \mu \epsilon$. This strain yields a theoretical BFS displacement of $10 \mathrm{MHz}$, as indicated by the vertical black line in Fig. 1(b). Computed values of BFS displacements in the case of Stokes-SSB, balanced DSB and unbalanced DSB are 13.7 $\mathrm{MHz}, 13.3 \mathrm{MHz}$, and $12.6 \mathrm{MHz}$, respectively. These three values thus overestimate the BFS displacement of $10 \mathrm{MHz}$ because of the pump depletion and pulse distortion, observed in Fig. 1(a). When using the anti-Stokes SSB probe regime, the BFS displacement was numerically calculated as $9.7 \mathrm{MHz}$ (continuous bold red curve in Fig. 1(b)), which is nearest to the expected value of $10 \mathrm{MHz}$. Thus the anti-Stokes SSB regime allows for the suppression of biases in sensing measurements.

\section{EXPERIMENTAL RESULTS}

To verify our numerical predictions, we set up the BOTDA sensor depicted in Fig. 2. In short, a distributed feedback (DFB) laser at $1535 \mathrm{~nm}$ is split into the pump and probe arms by a 50:50 tap coupler. In the pump side (fiber input), we insert an intensity modulator driven by a pulse generator to generate

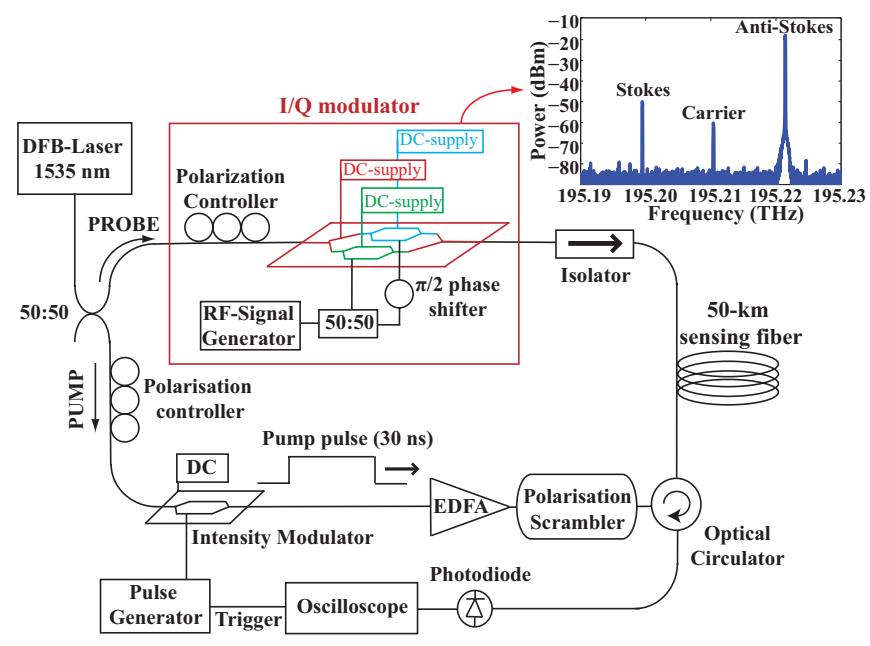

Fig. 2. Experimental setup of the Brillouin loss-based BOTDA sensor based on anti-Stokes single-sideband modulation technique.

square pump pulses with a width of $30 \mathrm{~ns}$, which provides a $3 \mathrm{~m}$ spatial resolution. These pulses are then amplified by an erbium doped-fiber amplifier (EDFA) and polarizationscrambled before being launched into a $50 \mathrm{~km}$ sensing fiber via an optical circulator. Our intensity modulator has a high extinction ratio of $40 \mathrm{~dB}$ which prevents anti-Stokes probe depletion due to the pump pulse leakage [10]. In the probe side (fiber output), we use an I/Q modulator [11] to generate an anti-Stokes probe whose frequency is swept around the BFS of the sensing fiber. More specifically, it consists in two parallel Mach-Zehnder interferometers (MZI) nested into a third combining MZI. An RF tone signal tunable around 11 $\mathrm{GHz}$ is injected into both RF ports of the modulator with a $\pi / 2$-phase shift. With this SSB-SC modulator, we achieved a carrier suppression of $45 \mathrm{~dB}$ and a side-mode suppression of $35 \mathrm{~dB}$ at $1535 \mathrm{~nm}$, as shown in the inset of Fig. 2. The probe power is set to $1.6 \mathrm{~mW}$ and injected into the sensing fiber after an isolator. At the fiber output, the anti-Stokes probe is extracted by a circulator and a 10-GHz bandpass FBG filter before being analyzed by a digital oscilloscope and an optical spectral analyzer (OSA). By sweeping the optical probe frequency, the BGS along the fiber is mapped as a function of frequency. In this way, all BFS variations along the fiber due to temperature change or applied strain can be detected. For the data acquisition, we have normalized the data with the DC offset by using the DC mode of our oscilloscope with an impedance of $1 \mathrm{M} \Omega$ to increase the photodetector sensitivity.

First, we performed distributed measurements without applying strain to compare the anti-Stokes SSB case to standard DSB technique using the same input power conditions. Figures 3(a) and (b) show the BOTDA traces in false color for the two regimes, respectively. From these figures, we plotted in Fig. 3 (c) the Brillouin gain (blue) and loss (red) amplitudes versus the distance for a frequency value of $10.925 \mathrm{GHz}$, and in Fig. 3(d), the Brillouin gain and loss spectra measured at the fiber end. The value of $10.925 \mathrm{GHz}$ actually corresponds to the Brillouin frequency shift of our fiber. It has been determined from a Lorentzian fit of the Brillouin loss spectrum plotted 

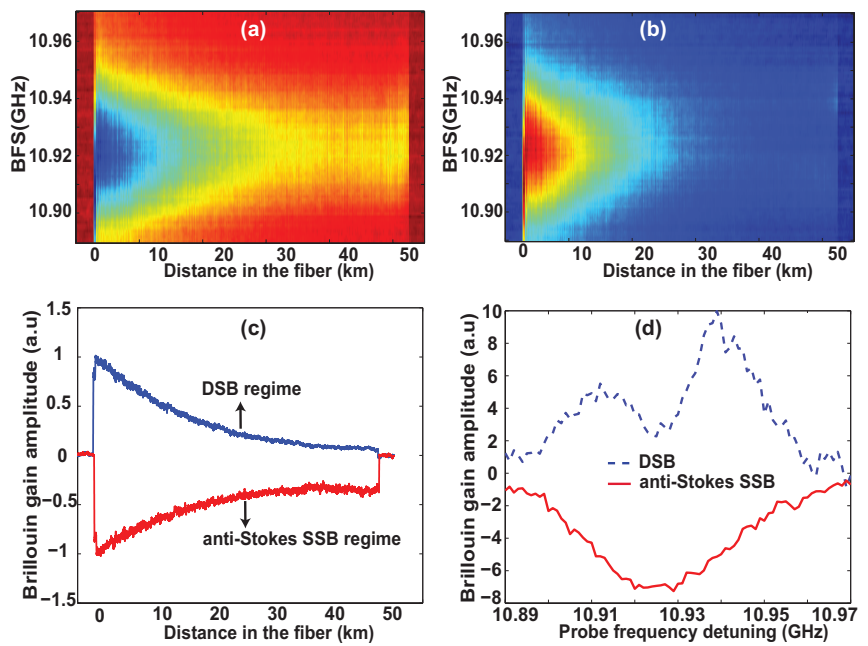

Fig. 3. Distributed measurements over a $50 \mathrm{~km}$ single-mode fiber using (a) the anti-Stokes SSB regime and (b) the balanced DSB regime. (c) Brillouin gain amplitude as a function of the fiber length for the two regimes measured at the Brillouin frequency shift of $10.925 \mathrm{GHz}$. (d) Brillouin gain amplitude as a function of the probe frequency detuning measured at the fiber end.

in red in Fig. 3(d). There are several conclusions we might draw from all these figures. First, as it can be seen in Fig. $3(\mathrm{c})$, the probe amplitude at the beginning of the fiber is very similar in both cases but the contrast at the fiber end is significantly improved by using the anti-Stokes probe. A gain in sensitivity can be clearly seen at the end of the fiber with the anti-Stokes SSB case (red) compared to the DSB case (blue). We measured an improvement of the SNR of about 5 times. Second, whereas the use of the anti-Stokes probe provides a symmetric and single-hump Brillouin loss spectrum, Fig. 3(d) shows that the Brillouin gain spectrum in blue exhibits an asymmetric double-humped shape, which leads to a BFS displacement, as numerically shown in Fig. 1(b). Clearly, this direct comparison demonstrates the detrimental effect of pump depletion in long-range measurements and, in particular, how the anti-Stokes probe can help to further reduce this issue.

Our BOTDA sensor system has then be tested with different strain elongations up to $66 \mathrm{~mm}$ over $24 \mathrm{~m}$ at the fiber output. The aim is to check that no bias or error has been introduced by the pump depletion with the SSB modulator. Fig. 4(a) shows the BFS displacement at the fiber end due to applied strain. We measure a maximum frequency shift of $35 \mathrm{MHz}$ that corresponds to a strain of $742 \mu \epsilon$. The BFS displacement versus applied strain is plotted in Fig. 4(b). We obtained by linear fitting a strain coefficient of $4.28 \mathrm{MHz} / 100 \mu \epsilon$, which is in quite good agreement with the value of $5 \mathrm{MHz} / 100$ $\mu \epsilon$ reported in literature [12]. This result means that errors introduced by the pump depletion in the anti-Stokes SSB regime can be ignored. Since the pump depletion effect is non-linear, the BFS displacements will also be non-linear if there are errors from the pump depletion. We also must stress here that we were not able to detect the applied strain when using the balanced DSB probe due to the very low SNR of our sensor at the end the fiber, as shown in Fig. 3(c). This also means that the SNR and thus the sensitivity has been improved with our technique compared to the balanced DSB case.
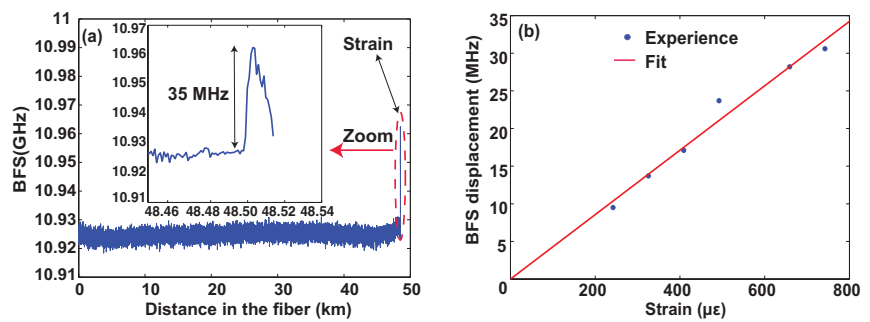

Fig. 4. (a) Brillouin frequency shift (BFS) with a strain of $742 \mu \epsilon$ over $24 \mathrm{~m}$ at the end of the fiber. Insets: zoom of the end of the fiber (b) Measurement of strain coefficient at the end of the fiber versus the applied strain.

\section{CONCLUSION}

We have made a long-range BOTDA sensing system that uses an anti-Stokes SSB-SC modulation technique. Enhanced sensitivity has been demonstrated both numerically and experimentally using this technique compared to the dual sideband standard one. This has been achieved by using a dual parallel Mach-Zehnder I/Q modulator in the probe path. Finally, our sensor has also been verified on a 50km-long SMF with a measured strain coefficient of $4.28 \mathrm{MHz} / 100 \mu \epsilon$. This work should also contribute to a further understanding of the main limitations in BOTDA sensors.

\section{REFERENCES}

[1] L. Thevenaz, S. F. Mafang and J. Lin, "Depletion in a distributed Brillouin fiber sensor: Practical limitation and strategy to avoid it," Proc. 21st Int. Conf. Opt. Fiber Sensors, (2011).

[2] A. Minardo, R. Bernini, L. Zeni, L. Thevenaz, and F. Briffod, "A reconstruction technique for long-range stimulated Brillouin scattering distributed fiber-optic sensors: experimental results," Meas. Sci. Technol., vol. 16, no. 4, pp. 900-908 (2005).

[3] M. A. Soto, G. Bolognini, and F. Di Pasquale, "Optimization of long-range BOTDA sensors with high resolution using first-order bidirectional Raman amplification," in Optics Express, vol. 19, no. 5, pp. 4444-4457 (2011).

[4] X. Angulo-Vinuesa, S. Martin-Lopez, J. Nuno, P. Corredera, J. AniaCastanon, L. Thévenaz and M. Gonzalez-Herraez, "Raman-Assisted Brillouin Distributed Temperature Sensor Over 100 km Featuring $2 \mathrm{~m}$ Resolution and 1.2 ○C Uncertainty," J. Lightwave Technol., vol. 30, pp. 1060-1065 (2012).

[5] Y. Dong, Li. Chen, and X. Baol, "Extending the Sensing Range of Brillouin Optical Time-Domain Analysis Combining Frequency-Division Multiplexing and In-Line EDFAs," J. Lightwave Technol., vol. 30, pp. 1161-1167 (2012).

[6] M. A. Soto, G. Bolognini, and F. Di Pasquale, "Long-range simplexcoded BOTDA sensor over $120 \mathrm{~km}$ distance employing optical preamplification," Optics Letters, vol. 36, no. 2, pp. 232-234 (2011).

[7] R. Bernini, A. Minardo and L. Zeni, "Long-range distributed Brillouin fiber sensors by use of an unbalanced double sideband probe," in Opt. Express, vol. 19, no. 24, pp. 23845-23856 (2011).

[8] X. Bao, D. J. Webb, and D. A. Jackson, "32-km distributed temperature sensor based on Brillouin loss in an optical fiber," Opt. Lett., vol. 18, pp. 1561-1563 (1993).

[9] M. J. Damzen, V. I. Vlad, V Babin and A Mocofanescu "Stimulated brillouin scattering: fundamentals and applications," IoP Publishing (2003).

[10] S. Afshar V., G. A. Ferrier, X. Bao, and L. Chen, "Effect of the finite extinction ratio of an electro-optic modulator on the performance of distributed probe-pump Brillouin sensorsystems," Opt. Lett., vol. 28, pp. 1418-1420 (2003).

[11] B. Stiller, M.W. Lee, J. Hauden, A. Mottet, D. M. Nguyen, H. Maillotte and T. Sylvestre, "Fiber optic Brillouin distributed sensing using phaseshift keying modulation techniques," Proc. SPIE, 8439-8 (2012).

[12] X. Bao, D. J. Webb, and D. A. Jackson, "Very high resolution optical spectrometry by stimulated Brillouin scattering," IEEE Photon. Technol. Lett., vol. 17, no. 4, pp. 855-857 (2005). 